\title{
Social optimal shifts and fares of taxi services
}

\author{
Josep Maria Salanova Grau \\ Associate Researcher, Centre for Research and Technology Hellas (CERTH) - Hellenic \\ Institute of Transport (HIT), Greece \\ Miquel Estrada \\ Assistant Professor, Center for Innovation in Transport (CENIT), Barcelona Tech, \\ Technical University of Catalonia (UPC), Spain
}

\section{SUMMARY}

This paper presents a mathematical model for supporting the decision and policy making related to the optimal determination of shifts and fares of taxi services in a major city. The model is based on the system's generalized cost function and uses continuous approximations and geometric probabilities for estimating the key performance indicators of the taxi market, which are waiting and access time for the customers (in-vehicle travel time does not depend on the offer side) and benefits for the drivers. The model is based on an econometric model with the inclusion of an elastic demand, which allows the estimation of the optimal values for the two decision variables of the problem: fare structure and the taxi fleet size. The model also accounts for a full-day time period instead of the one-hour time frame models used in most of the taxi models, which allows for providing insights on the daily duration and distribution of the shifts that should be defined by the policy makers.

\section{INTRODUCTION TO TAXI POLICY REGULATION AND DEREGULATION}

Taxis are an individual transport mode used for public transport services providing door to door personal transport services. They can be divided into three broad exploitation categories: stand, hailing and dispatching market. They differ in the way the taxis services are offered to the customers. In the taxi stand, taxi stops are used as a meeting point between taxis and customers through a FIFO (First-in-First-out) queueing system. In the hailing market, customers hail a cruising taxi on the street. In this case, there is high uncertainty for both the drivers, which do not know the percentage of vacant kilometers, and the customers, which are not aware of the waiting time and the quality/fare of the service they will find. In the dispatching market, customers call a dispatching center requesting for an immediate taxi service. Only in this kind of market consumers can choose between different service providers or companies. At the same time, companies can fidelize customers by providing good quality services or billing advantages. It is observed that each category performs better depending on the social characteristics of the city as well as the potential demand to be served or the policy applied by the decision makers, having significant impact on the profitability of the sector, the level of service offered and the externalities generated. There is a need for regulating the taxi service in each city in order to reduce the negative impacts and externalities created to the other drivers and citizens by the taxi sector such as congestion or emissions. 
The aim of this paper is to present a model able to assess the decision making related to the taxi policy in urban regions considering shifts and fares as a policy tools. The proposed model uses the different mathematical formulations presented in the literature for estimating the optimum fleet size related to each operational mode, city size and demand level, the fare policy to be applied as well as the taxi shifts policy that should be applied, which will allow for defining the optimum regulation measures.

The paper is organized as follows: the state of practice regarding taxi policy making is presented in the second section; the formulation of the model for taxi services with elastic demand is presented in the third section together with the methodology for optimizing the shift duration and distribution along the day. Finally, section six presents the conclusions of the above analyses.

\section{STATE OF ART}

The main discussion of how to fix the fare for taxi services is related to choosing the first best solution, which is related to the social optimum where the system unitary costs is minimum (Cairns and Liston-Heyes, 1996; Arnott, 1996; Yang et al., 2002; Fernandez et al., 2006; Chang and Chu, 2009) or to the second best solution, which is related to the zero-profit of drivers where the system unitary costs is minimum without generating negative benefits to the drivers (Cairns and Liston-Heyes, 1996; Yang et al., 2002; Fernandez et al., 2006; Chang and Chu, 2009). Regarding the demand estimation, few models use an elastic function for the estimation of the demand for taxi services (Wong et al., 2001; Wong and Wong, 2002). Salanova et al. (2011) presents an overview of these models. Finally, regarding the shifts policy, to the knowledge of the authors no methodologies have been proposed since all the modeling efforts are related to the optimization of the total number of offered taxi-hours mostly focusing on peak hours, without taking into account how these are distributed during the day within the peak and off-peak hours.

\section{MODELING FOR TAXI POLICY}

The econometric model used in this paper is based on the system unitary cost for the provision of the taxi services, accounting for the users' costs, the drivers' benefits (seen as a negative cost) as well as the externalities generated by the taxis. All costs are estimated using continuous approximations and geometric probabilities while the optimization is done analytically. Salanova et al. (2014) review various formulations developed for the estimation of the variable of the taxi market, some of them used in the model. The model is presented in detail in Salanova (2013) and is complemented with the following three additions: elastic demand; $24 \mathrm{~h}$ demand profile and shifts policy.

The proposed objective function, which applies to the three operational modes, is presented in Equations (1)-(5). Table 1 summarizes the model inputs and decision variables. It is worth to note that the trip monetary cost is cancelled in the objective function but is kept in 
the formulations since it constraints the benefit of the drivers and therefore the feasibility of the provision of taxi services form the operator point of view.

$\operatorname{Min} Z=Z_{d}+Z_{u}+Z_{c}+G$

$Z_{u}=\lambda_{u} \cdot A \cdot\left[\alpha_{A} \cdot T_{A}+\alpha_{W} \cdot T_{W}+\alpha_{I V} \cdot T_{I V}+\frac{\bar{c}}{V o T_{u}}\right]$

$Z_{d}=\frac{\lambda_{d} \cdot A}{V o T}\left[-\bar{n} \cdot \bar{c}+\left(\bar{d} \cdot C_{k m}+C_{h}\right)\right]$

$Z_{c}=\lambda_{v} \cdot A \cdot \Delta T_{v}+\frac{\lambda_{d} \cdot A \cdot C_{E} \cdot E_{d}}{V o T}+\frac{\lambda_{v} \cdot A \cdot C_{E} \cdot \Delta T_{v} \cdot E_{d}}{V o T}$

$G=C_{s} \cdot n_{s}$

The access, waiting and in-vehicle time values are calculated following the formulations proposed in Salanova (2013). In addition, a set of constraints is proposed for reflecting physical or temporal restrictions of the policy decisions, which are related to the market goals. The values that should be within acceptable thresholds are the access and waiting time of customers, the benefit of taxi drivers, the emissions and congestion levels, the infrastructure costs and the number of licenses.

\begin{tabular}{|c|c|}
\hline \multicolumn{2}{|c|}{ Variable } \\
\hline \multirow{10}{*}{ 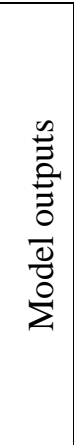 } & $\mathrm{Z}$ is the cost of the system $(\min )-\mathrm{z}$ is the unitary system cost $(\mathrm{min} /$ trip $)$ \\
\hline & $Z_{d}$ is the cost of the drivers (min) $-z_{d}$ is the unitary cost of the drivers (min/trip) \\
\hline & $Z_{u}$ is the cost of the customers ( $\left.\min \right)-z_{u}$ is the unitary cost of the customers (min/trip) \\
\hline & $Z_{c}$ is the additional cost for the city ( $\left.\min \right)-z_{c}$ is the unitary cost for the city (min/trip) \\
\hline & $G$ is the cost of the infrastructure ( $\min )-g$ is the unitary infrastructure cost ( $\mathrm{min} /$ trip) \\
\hline & $T_{W}$ is the waiting time of customers ( $\mathrm{min}$ ) \\
\hline & $T_{A}$ is the access time of customers (min) \\
\hline & $\bar{c}$ is the average trip cost $(€)$ \\
\hline & $\bar{n}$ is the average number of trips per hour and driver (trips) $=\lambda_{u} / \lambda_{d}$ \\
\hline & $\Delta T_{v}$ is the increase in the travel time of the other drivers caused by taxis (min) \\
\hline \multirow{3}{*}{ 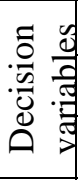 } & $\lambda_{d}$ is the taxi hourly supply (vehicles per hour and area of service) \\
\hline & Variables related to the calculation of the trip cost (flag drop, fee per unit of distance) \\
\hline & $\mathrm{n}_{\mathrm{s}}$ is the number of stands \\
\hline \multirow{7}{*}{ 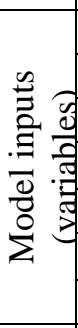 } & $\lambda_{\mathrm{u}}$ is the hourly demand for taxi trips (trips per hour and area of service) \\
\hline & $\mathrm{A}$ is the area of the region $\left(\mathrm{km}^{2}\right)$ \\
\hline & $\lambda_{v}$ is the hourly circulating vehicles (vehicles per hour and area of service) \\
\hline & $\bar{d}$ is the average distance run by each taxi in one hour $(\mathrm{km})=0.5 \mathrm{~A}$ \\
\hline & $\overline{\mathrm{v}}$ is the average speed of the trip $(\mathrm{km} / \mathrm{h})$ \\
\hline & $T_{I V}$ is the in-vehicle time of customers $(\min )=\bar{d} / \overline{\mathrm{v}}$ \\
\hline & $E_{d}$ is the hourly vehicle emissions $\left(\mathrm{kg}\right.$ of $\left.\mathrm{CO}_{2}\right)$ \\
\hline \multirow{8}{*}{ 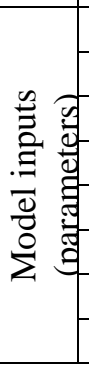 } & $\operatorname{VoT}_{u}$ is the value of time $(€ / \mathrm{min})$ \\
\hline & $\alpha_{A}$ is the customer perception factor of the access time \\
\hline & $\alpha_{W}$ is the customer perception factor of the waiting time \\
\hline & $\alpha_{I V}$ is the customer perception factor of the in-vehicle time \\
\hline & $C_{S}$ is the hourly cost of each taxi stand $(€ / \mathrm{min})$ \\
\hline & $C_{k m}$ is the operational cost per unit of distance of taxis $(€ / \mathrm{km})$ \\
\hline & $C_{h}$ is the hourly operational cost of the moving taxis $(€ / \mathrm{min})$ \\
\hline & $C_{E}$ is the emission unitary cost for all vehicles $\left(€ / \mathrm{kg}\right.$ of $\left.\mathrm{CO}_{2}\right)$ \\
\hline
\end{tabular}

Table 3 Definition of the variables of the model and the reference values 
Two main improvements have been done to the dispatching model presented in Salanova (2013) in order to illustrate its use for the taxi policy making:

- The model has been upgraded for the purpose of this paper introducing elastic demand in order to be able to take into account the effect of the pricing policy in the demand. In the original model, the impact of the pricing is internal, since the monetary cost of the trip applies to the customers and the drivers with opposite sign, having no impact in the system cost. This internal cost has an impact on the viable number of taxis from the driver point of view, but not in the demand for taxi trips (and therefore in the second best only, not in the first best).

- In addition, an optimization model has been developed for adjusting the real supply based on shifts to the optimum supply obtained applying the econometric model.

\subsection{Impact of pricing in the taxi model}

An exponential demand function of the customers' utility similar to the one proposed by Wong et al. (2001) is used in order to analyze the impact of the pricing policy (Equation 6). The utility function of the users is composed by the waiting time and the trip monetary cost calculated as a flag-drop and the trip distance multiplied by a kilometric cost in temporal units.

$$
\lambda_{u}=\widetilde{\lambda_{u}} \cdot e^{-\gamma\left(\alpha_{W} \cdot T_{W}+\frac{D+\bar{d} \cdot \tau_{k m}}{V o T_{u}}\right)}
$$

where,

$\widetilde{\lambda_{\mathrm{u}}}$ is the maximum demand for taxi trips

$\gamma$ is the scaling parameter

$\alpha_{W}$ is the customer perception factor of the waiting time

$\mathrm{T}_{\mathrm{W}}$ is the waiting time of customers ( $\mathrm{min}$ ) calculated as in Salanova and Estrada (2015)

$D$ is the flag-drop charge $(€)$

$\overline{\mathrm{d}}$ is the average distance of the trip $(\mathrm{km})$

$\tau_{\mathrm{km}}$ is the taxi fee per unit of distance $(€ / \mathrm{km})$

$\operatorname{VoT}_{\mathrm{u}}$ is the value of time of the taxi customers $(€ / \mathrm{min})$

The fact that the demand depends on the waiting time and, at the same time the waiting time depends on the demand creates the necessity for an iterative bi-level optimization problem, where the optimum fleet is calculated at the upper level by minimizing the unitary system costs while the waiting time related to this supply and the demand related to this waiting time are calculated at the lower level. The bi-level problem is solved by selecting an initial supply level (number of taxis) and solving the lower level problem, whose solution (new demand) is used in an iterative process until equilibrium is reached. Waiting time is the only user cost component that depends on the provision of taxi service (supply). Therefore, this user component needs to be updated every time the supply density of taxi cabs is changed. 


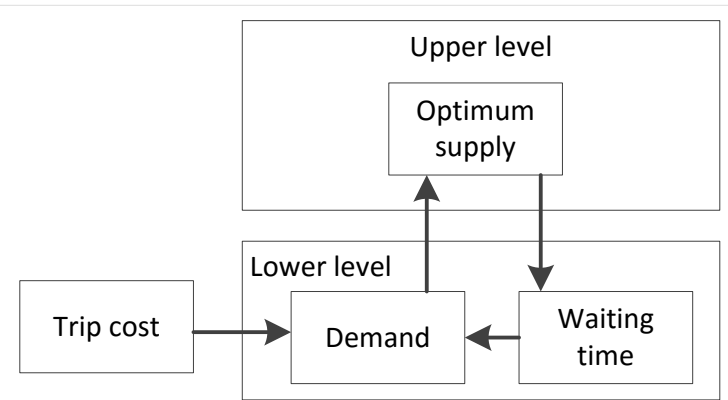

\section{Figure 1 Bi-level optimization problem with elastic demand}

Applying the elastic demand formulation to the econometric model and solving the bi-level problem, Figure 2 is obtained, which depicts the convergence of the total demand and waiting time as the number of iterations increase. The bi-level solution is obtained by solving independently and iteratively/sequentially each one of the two problems (using an initial solution for the variable of the lower level as starting point) until convergence of the solution is obtained. The results of the optimization problem are independent of the initial waiting time value, as shown with the convergence of the values in Figure 2. This fact gives robustness to solution obtained from the proposed bi-level problem.

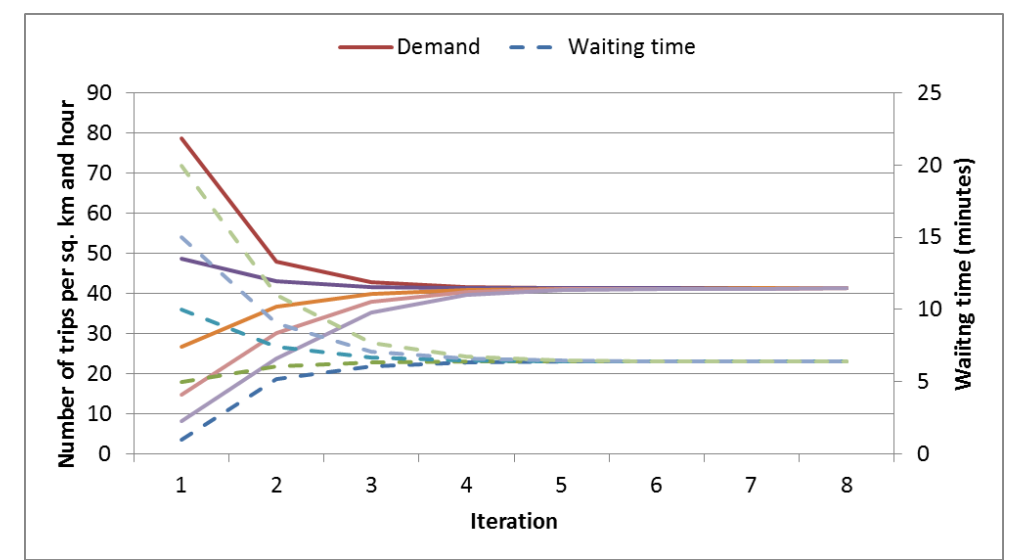

Figure 2 Convergence of the demand and the waiting time

\subsection{Adaptation of the optimal fleet size to the shifts policy}

Most of the taxi markets around the world have regulated the working days of the taxis in order to regulate the externalities produced, while protecting the drivers themselves. These regulations are provided as feasible working days in a weekly basis or in the form of working shifts in a daily basis, which can have various durations, so the taxi drivers should adapt their working hours to the feasible slots provided by the regulating authorities. The optimum taxi fleet size of the above model is quantified in vehicle-hours per hour, and policies and regulations should be applied aiming at having the right number of vehicle hours at each hour of the day. In order to do this the model is applied to the 24 hourly intervals of taxi demand, obtaining 24 taxi supply levels, one for each hour. The difference between the supply level provided by shifts policy and the optimum supply level can be easily measured as the area between the two curves. An example is presented in Figure 3. 
The optimal curve is obtained by applying the model to each hour of the day, while the real supply is obtained by adding the number of taxis in the streets at each hour of the day taking into account that each taxi will work for the whole duration of the shift. Shorter shifts will generate real curves with a better fit (compared to the optimal) than larger shifts. The optimum shift would have a duration of one hour.

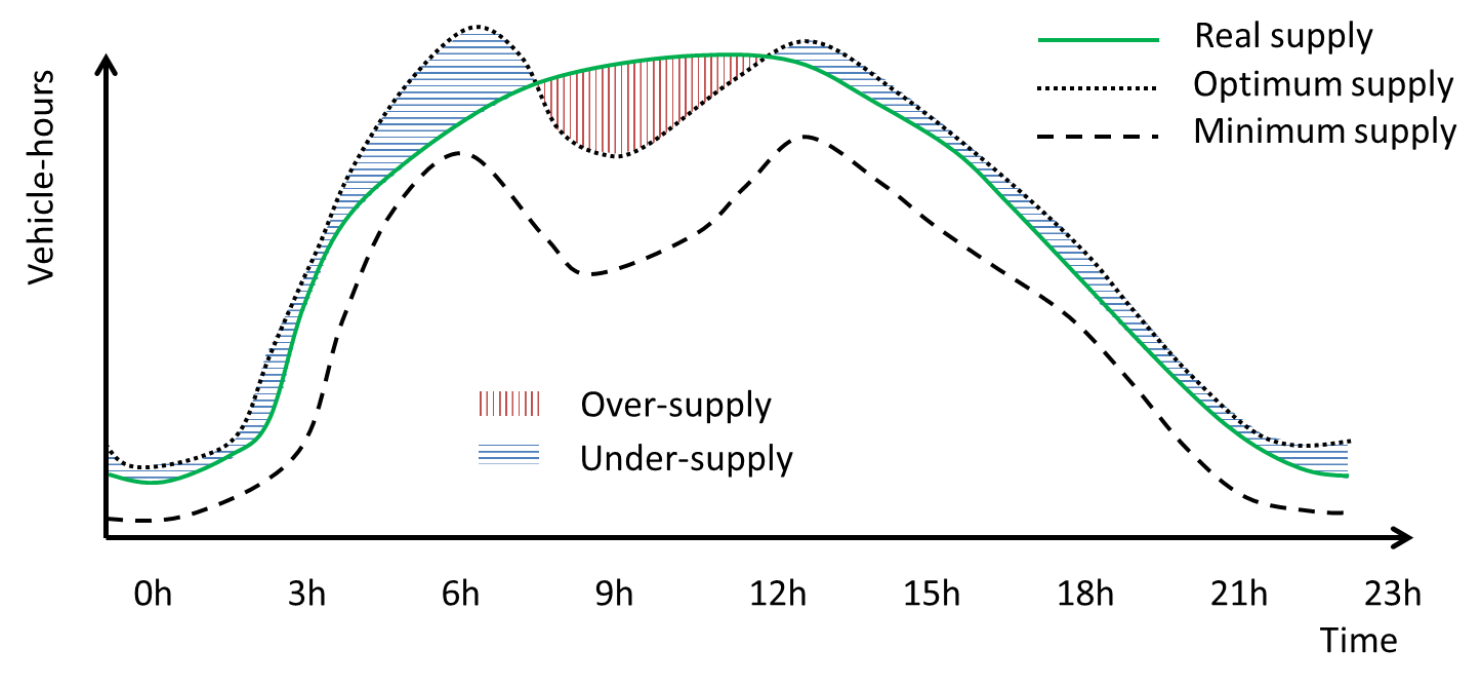

Figure 3 Real, optimum and minimum supply during the day

The impacts of the differences between the two curves can be estimated by using the formulations presented in the proposed model. They are basically the increase/decrease of waiting time of customers and the increase/decrease in the benefits of taxi drivers. More taxis than the optimum value will reduce the waiting time but will also reduce the benefits of the taxi drivers. In comparison, less taxis than the optimum value will increase the benefits of the taxi drivers but, at the same time, will increase the waiting time of customers.

An optimization algorithm has been developed for adapting the shifts policy to the results obtained by the model. The algorithm solves an optimization problem defined in Equations (7)-(10). It is a square error minimization problem between the optimum $\left(\mathrm{O}_{\mathrm{i}}^{*}\right)$ and the real provided taxi-hours $\left(\mathrm{AS}_{\mathrm{i}}\right)$ during each hour (i) of the day for each shift duration based on the number of taxis starting their shift at each hour $\left(\mathrm{S}_{\mathrm{i}}\right)$.

Subject to:

$$
\min \sum_{i=1}^{24}\left(O_{i}^{*}-A S_{i}\right)^{2}
$$

$$
\begin{gathered}
A S_{i}>\overline{O_{i}} \\
\sum_{i=1}^{24} S_{i}=M *(L / l) \\
S_{i}>0 \forall i=1: 24
\end{gathered}
$$


where,

$\mathrm{O}_{\mathrm{i}}^{*}$ is the optimum number of taxi-hours during hour $\mathrm{i}$

$\mathrm{AS}_{\mathrm{i}}$ is the number of taxi-hours during hour $\mathrm{i}$

$\overline{\mathrm{O}}_{1}$ is the minimum number of taxi-hours during hour $\mathrm{i}$

$\mathrm{S}_{\mathrm{i}}$ is the number of taxis starting their shift at hour $\mathrm{i}$

$\mathrm{M}$ is the maximum daily number of taxis working

$\mathrm{L}$ is the maximum daily working hours per driver

$\mathrm{l}$ is the duration of the shift

A is the shift matrix, where $A_{i j}=\left\{\begin{array}{c}1 \text { if } i \leq j \leq i+1 \\ 0 \text { elsewhere }\end{array}\right.$

Constraint (8) assures that the demand for taxi trips will be satisfied. The minimum supply $\left(\mathrm{O}_{\mathrm{i}}^{*}\right)$ is obtained from the econometric model. Constraint (9) obliges that all taxis will work during their whole set of shifts respectively while Constraint (10) is a non-negativity constraint.

\section{CONCLUSIONS}

Two issues related to the taxi policy have been analyzed in this paper, the inclusion of demand elasticity and the adaption of the taxi shifts policy to the optimum supply obtained by the aggregated model. The first one is related to the fare regulation and the limitation of licenses, more related to planning issues while the second one is related to the daily operation of the taxi shifts. Both issues are of crucial importance for the provision of high-quality taxi services, especially nowadays with the introduction of alternative service providers (uber, car sharing, bike sharing...), and this paper provided a research framework that can be put in practice in order to benefit both the taxi drivers and user as well as the citizens in general.

The impact of pricing on the demand and the Level of Service has been provided by defining an elastic demand function. The optimum combination of fare and fleet size have been identified, providing a small number of taxis and high fares as optimum for the drivers, and a large number of taxis with low fares as optimum for the customers. The customers are more sensible to the waiting time for low taxi densities, where the fare has small impact on the demand. Oppositely, for large fleets, the waiting time is not the constraining factor that customers take into account, as opposed to the monetary cost of the trip itself.

From the above, it can be concluded that there is a need for regulating the taxi sector both in terms of fare and number of vehicles from the planning perspective, but also there is a strong need for regulating the shifts policy in order to achieve a better operational framework in which all the involved actors (drivers and users) will be beneficiated. 


\section{ACKNOLEDGEMENTS}

The authors would like to thank the Center for Innovation in Transport (CENIT) for the provision of the data. This work was supported by the Project ENTROPIA from the Spanish "Ministerio de Economía y Competitividad". Proyectos de Investigación Fundamental No Orientada. REF: TRA2012-39466-C02-01.

\section{REFERENCES}

AMAT I BERTRAN C. (2010) Anàlisi de l'eficiència del servei de taxi a Barcelona. Propostes de millora. Tesina final de Carrera. ETSECCPB Barcelona.

ARNOTT R., (1996) Taxi Travel Should Be Subsidized. Journal of Urban Economics $40,316-333$.

CAIRNS R. D., Liston-Heyes C. (1996) Competition and regulation in the taxi industry. Journal of Public Economics 59, 1 - 15.

CHANG S. K. AND CHU-HSIAO CHU (2009) Taxi vacancy rate, fare and subsidy with maximum social willingness-to-pay under log-linear demand function. Transportation Research Record: Journal of the Transportation Research Board 2111, 90 - 99.

FERNANDEZ L. J. E., DE CEA CH. J. AND BRIONES M. J. (2006) A diagrammatic analysis of the market for cruising taxis. Transportation Research Part E 42, 498 - 526.

INSTITUT METROPOLITÀ DEL TAXI (IMT), www.taxibarcelona.cat

SALANOVA J. M., ESTRADA M., AIFADOPOULOU G. AND MITSAKIS E., (2011). "A review of the modeling of taxi services". Procedia and Social Behavioral Sciences 20: 150-161.

SALANOVA J. M., ESTRADA M., AMAT C. (2014). "Aggregated modeling of urban taxi services". Procedia - Social and Behavioral Sciences (ISSN: 18770428), 20: 352-361.

SALANOVA J. M. (2013). Modeling of taxi cab fleets in urban environment. PhD thesis, Polytechnic University of Catalonia, BarcelonaTECH.

WONG K. I., WONG S. C. AND YANG H. (2001) Modeling urban taxi services in congested road networks with elastic demand. Transportation ResearchPart B 35, 819 842 .

WONG K. I., WONG S. C. AND YANG H. (2001) Modeling urban taxi services in congested road networks with elastic demand. Transportation Research Part B 35, $819-$ 842.

YANG H., WONG S. C. AND WONG K. I. (2002) Demand-supply equilibrium of taxi services in a network under competition and regulation. Transportation Research Part $B$ $36,799-819$. 